\title{
THE PASCHEN DISCONTINUITY IN B STARS
}

\author{
ADELA E. RINGUELET* \\ Institut d'Astrophysique de l'Université de Liège, Belgique, and \\ Observatorio Astronómico de la Universidad de La Plata, Argentina
}

(Received 2 June, 1969)

\begin{abstract}
Measurements of the Paschen discontinuity in stars with $T_{\text {eff }} \geqslant 10^{4} \mathrm{~K}$ leads to the conclusion that the $D_{P} / D_{B}$ ratio increases with temperature faster than expected. The increase of $D_{P} / D_{B}$ with $(\log g)^{-1}$ is also steep.

Résumé. Les mesures de la discontinuité de Paschen dans les étoiles de $T_{\text {eff }} \geqslant 10^{4} \mathrm{~K}$ nous montrent que le rapport $D_{P} / D_{B}$ augmente avec la température plus rapidement que prevu. L'augment de $D_{P} / D_{B}$ avec $(\log g)^{-1}$ est aussi remarquable.
\end{abstract}

\section{Introduction}

The energy distribution in the continuum of main sequence stars is fairly well represented by the results of computations under L.T.E. hypothesis. As we move into the region of stars with lower surface gravity physical conditions become not so well established. With the aim of providing observational values which could be useful to any attempt of dealing with this problem and of discussing what has already been done, we determined Paschen discontinuities $\left(D_{P}=\lg I_{8200^{+}}-\lg I_{8200^{-}}\right)$for a group of stars with $T_{\text {eff }}>10^{4} \mathrm{~K}$.

\section{Material}

Among bright stars $\left(m_{v} \leqslant 6.0\right)$ of spectral types ranging from $\mathrm{O}$ to $\mathrm{A} 0$ and luminosity classes $\mathrm{V}$ to Ia, 55 stars were selected, 27 of them showing emission lines. Spectrograms were taken in $39 \mathrm{~A} / \mathrm{mm}$ with the $193-\mathrm{cm}$ reflector at Haute Provence. All data concerning the stars and plates are collected in columns 1 to 5 of Table I.

\section{Corrections and Reductions of the Observational Material}

The first intermediate results are the values of the continuum as obtained from the microphotometric tracings; to reduce them it appears that two corrections should be applied, namely, those that take into account,

a) the atmospheric absorption,

b) the variations in sensitivity with wavelength of the $1 \mathrm{~N}$ emulsion.

On account of the results by Mianes for the site at Haute Provence (Mianes, 1963) and the fact that our observations were made close to the zenith, the differential correction for atmospheric absorption in the region we are interested in, turned out

* Member of the Carrera del Investigador, Consejo Nacional de Investigaciones Científicas y Técnicas, Argentina. 


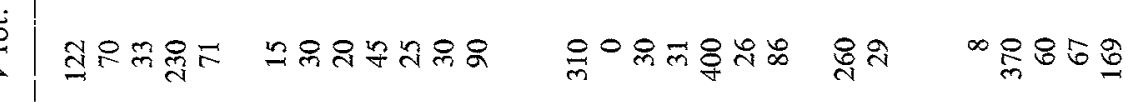

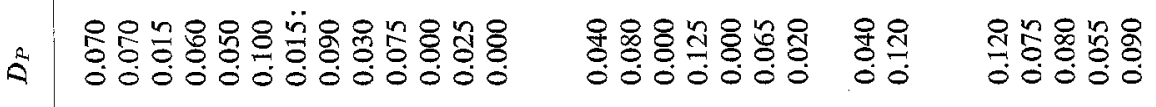

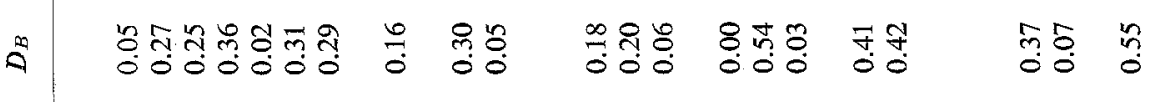

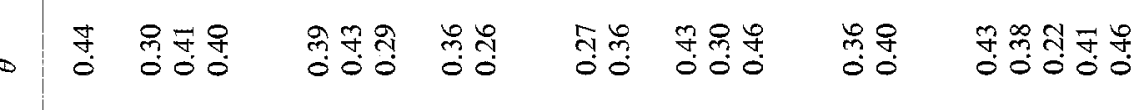

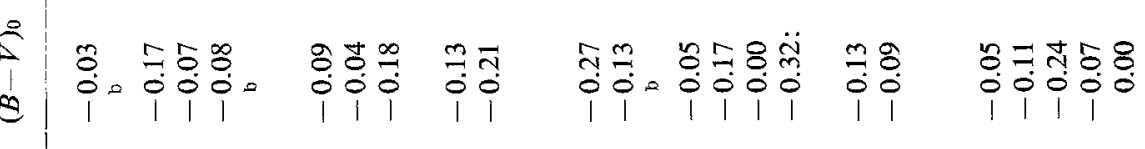

点

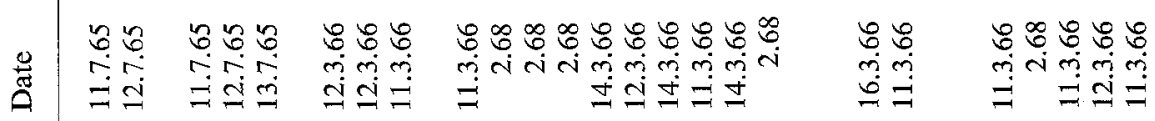

๕.

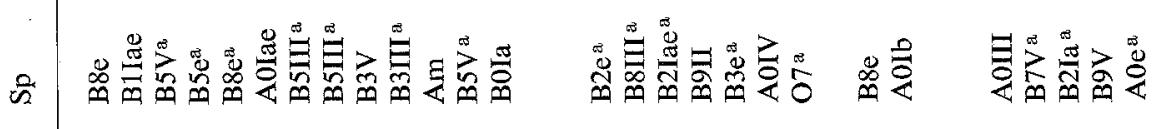

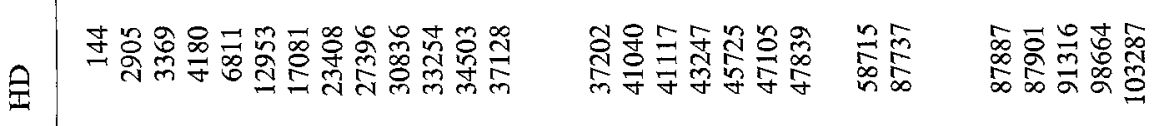

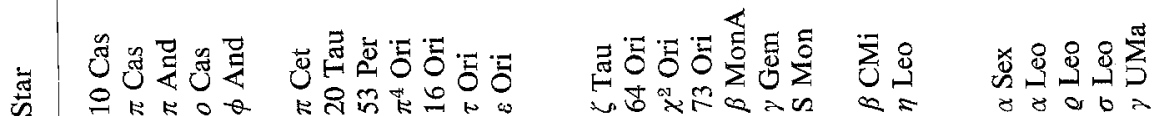




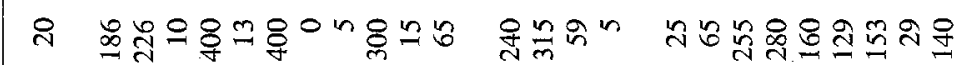

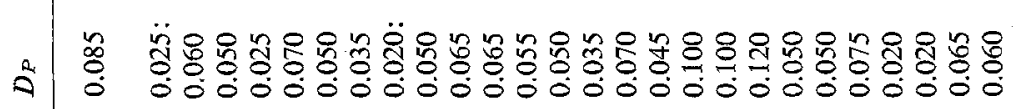

\&

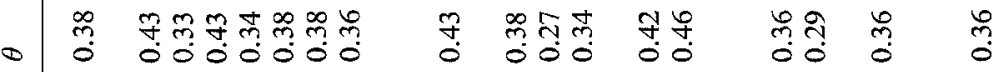

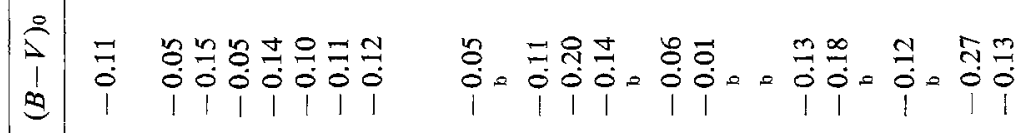

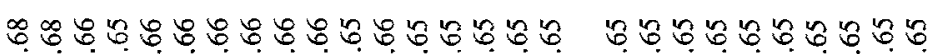

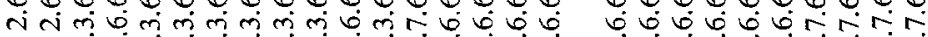

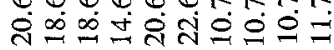

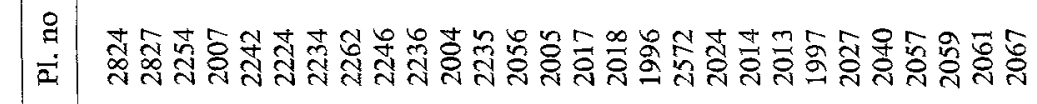

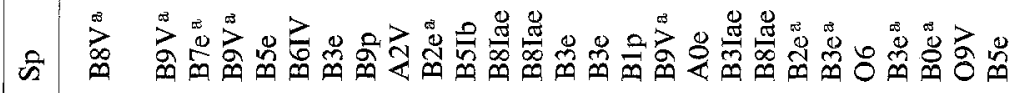
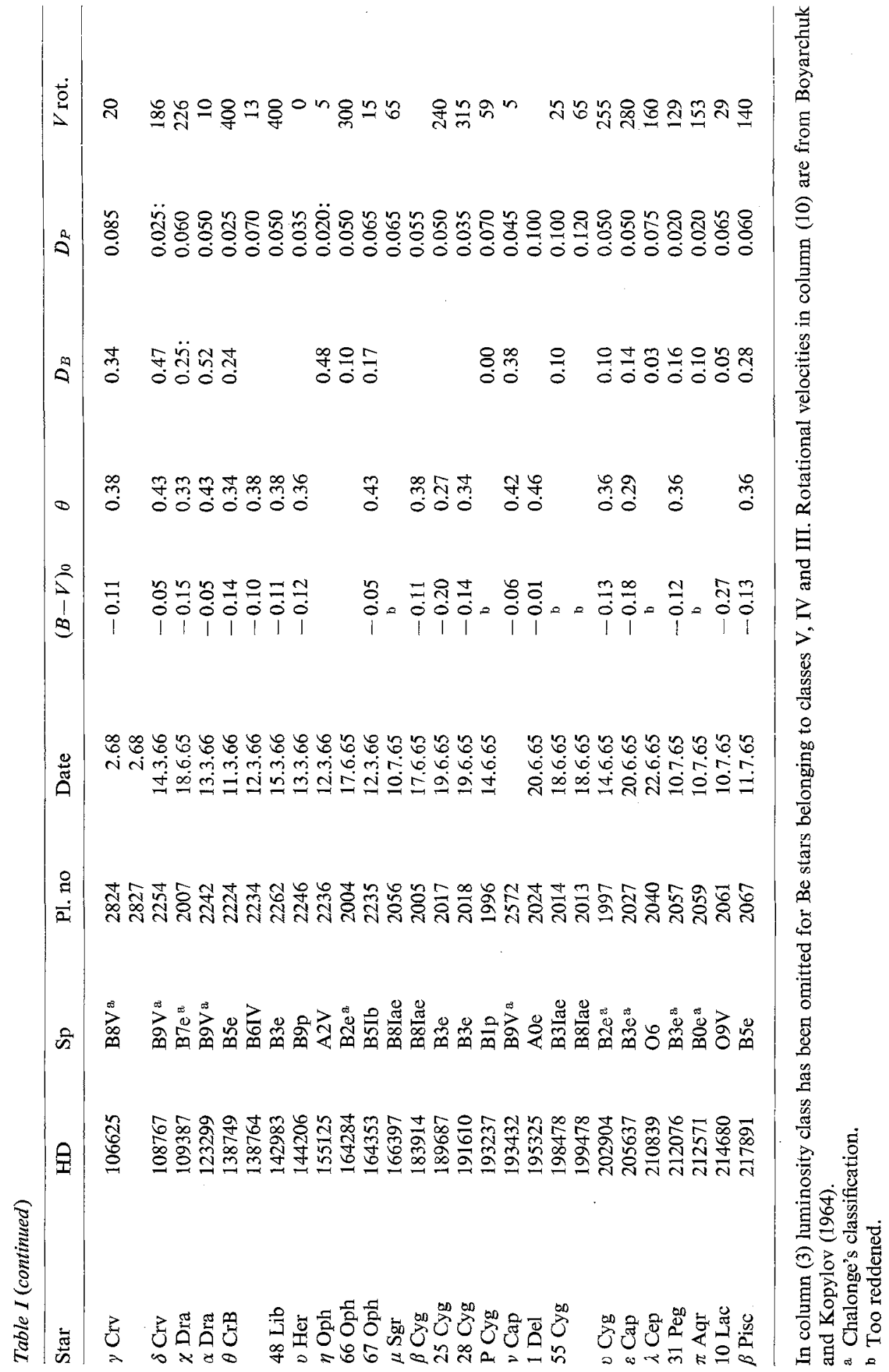
to be less than $0.006 \mathrm{mag}$. In spite of this an independent test for atmospheric absorption was carried out with plates of S Mon, taken at different hour angles, which were kindly obtained and generously put at my disposal by Mme. Andrillat. We verified that it was possible to select regions which were not differentially affected by this factor, at least within the range of observational errors. These regions were large enough so as to allow the determination of the slope of the continuum at both sides of the Pascken discontinuity. The adopted intervals were, in wavelength

$$
7450 \leqslant \lambda \leqslant 7500 ; \quad 7700 \leqslant \lambda \leqslant 8100 ; \quad 8300 \leqslant \lambda \leqslant 8700 .
$$

This result was further confirmed in a simultaneous check for variations in sensitivity with wavelength of the emulsion, with the stars $\gamma$ Crv and $\varepsilon$ Ori and 16 Mon included in Oke's photometry (Oke, 1964; Oke and Conti, 1966).

The relevant problem was the loss of sensitivity of the IN emulsion for wavelengths larger than $\lambda 7950$. The emulsion behaviour was checked on plates of 16 Mon with different density levels. We found that it was necessary to build three different correction curves, depending on the mean value of the transmission factor for the continuum,

i) $T c_{\lambda 8600} \leqslant 0.250$,

ii) $0.250<T c_{\lambda 8600}<0.350$,

iii) $T c_{\lambda 8600} \geqslant 0.350$.

We further tested these results by determining the Paschen discontinuity in $\alpha$ Leo and $\varepsilon$ Ori - studied by Oke (1964) - and $\gamma$ UMa and $\gamma$ Gem, studied by Mlle. Bloch and Mao-Lin (1956).

In conclusion, all of our measurements of the continuum are given relative to 16 Ori. No special correction for atmospheric absorption is included, but we were very careful about sensitivity corrections of the IN plates, as these turned out to be dependent of the density. In Figure 1 we present a comparison between our measurements and the ones by the authors mentioned above.

The Paschen discontinuity, as compared to the Balmer discontinuity, is much more masked by the overlapping of the wings of the absorption members of the series and therefore, a criterion had to be adopted in order to give a numerical value. The

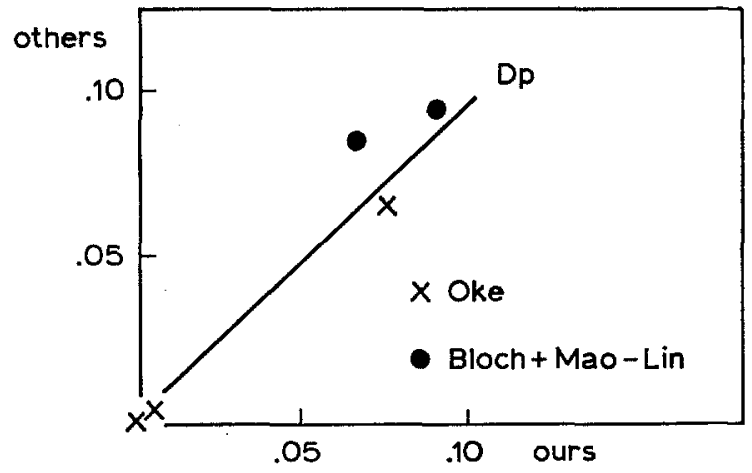

Fig. 1. 
procedure chosen was to represent the continuum in the interval $7450 \leqslant \lambda \leqslant 7850$ (interval $a$ ) by a straight line; on the other side of the Paschen jump we selected the region where the 'Paschen depression' appears to end (interval $b$ ) and use it for a second determination of the continuum, drawing another straight line. The value of our Paschen discontinuity corresponds to the difference at $I_{\lambda 8200}$ defined by the two straight lines.

$D_{P}$ depends upon the determination of levels and gradients in the continuum; we must control, somehow, the behaviour of these two elements. As far as we know, no gradients are available for this region, except for the ones which could be deduced from Oke's photometric work. From his measurements, gradients in region $a$ and $b$ $-\nabla a$ and $\nabla b-$ seem to differ slightly and always in the same sense, as ours do. The stars we have in common reproduce the figures and the whole set of values fit with the few points obtained from his data. We checked for any kind of correlation between $D_{P}$ and $\nabla$ and we found that the only correlation present is the one expected from the running of $\nabla$ with $(B-V)_{0}$ for main sequence stars.

\section{Masking of the Data}

Before presenting our results we must consider the factors that tend to mask the real value of the discontinuity. Even though they are of different nature we will discuss them together,

a) presence of emissions in the Paschen series,

b) extent of the absorption wings,

c) presence of companions to the stars - binary systems.

Some of the Be stars included in our list have the Paschen series in emission and among these a few show emission in the region towards the violet of the Paschen jump. The presence of emission in the lines does not influence our measurements as in most cases the absorption wings do not disappear completely and the same appears to be true for the continuum; a comparison with normal stars renders improbable that this emission could extend further than $\lambda 7700$ (at least in the cases we handled).

As to the effect of the absorption wings we verified that an elementary line profile for $P_{12}$, computed by considering only radiation damping, overlaps with the neighbouring lines when $I=0.90 I_{\text {continum }}$, so it appears necessary to introduce a correction to raise the continuum at the long wavelength side of the discontinuity. This correction may amount, taking into account the influence of the Stark effect, up to $30 \%$ of the original value for luminosity class $V$ stars. We do not know whether this consideration applies to Be stars. Even though these objects deserve a thorough discussion we will not include it here as the different suitable corrections do not modify the general tendency of the figures in Tables II and III. We feel that in no case the correction should be larger than $10 \%$.

In regard to the presence of binary components, we found three stars included in Batten's catalogue (1967) and two in his list of rejected objects; their value for the discontinuity falls very close to the mean value for their group. Moreover, considering 
the objects that may be double systems, we verified that there is no systematic difference with the rest.

\section{Results}

We grouped stars by spectral type and luminosity class to show - in the following tabulation - the mean value and the corresponding mean errors of the Paschen discontinuity, without including correction $4 \mathrm{~b}$.

We may note that the scatter of individual values relative to the mean is of the same amount as the scatter of the Balmer discontinuity values - as derived from the work by Chalonge and Divan (1952). Individual values are given in Table I, column 9; the corresponding error of one determination is \pm 0.005 .

From Table II it appears that $D_{P}$ decreases, within one luminosity class, with increasing temperature as it is expected from the behaviour of the continuum absorption coefficient of hydrogen. What does not appear so simple to understand are the variations related to luminosity class. If we introduce correction $4 \mathrm{~b}$, we obtain, the data given in Table IIa.

TABLE IIa

Corrected values of $D_{P}$

\begin{tabular}{lllll}
\hline Spectral range & \multicolumn{4}{l}{ Luminosity class } \\
& V & Be & III & SG \\
\hline A0-B5 & 0.061 & 0.067 & 0.094 & 0.099 \\
B5-B0 & 0.039 & 0.040 & 0.075 & 0.053 \\
$>$ B0 & & 0.053 & & \\
\hline
\end{tabular}

We cannot be sure whether or not there is a real difference between main sequence and Be stars - we cannot establish, if it does exist, the effect of the envelope - but, anyway, the general tendency we observed before remains true.

In order to compare our observational results with the ones theoretically predicted we need to assign effective temperatures and these were obtained through the colours. The $(B-V)$ adopted are from the Lunar and Planetary Lab. Publ. No. 63 corrected for interstellar reddening in the following way: we plotted $(B-V)_{0}$ against spectral types from Arp (1958). In the same plot we represented our stars with the observed $(B-V)$ and then we shifted the set of points to the left, as much as possible, thus providing a value for $E(B-V)$. The best representation of our stars, in relation to Arp's curve, was obtained with $E(B-V)=0.11 \mathrm{mag} / \mathrm{kpc}$, a value which is admissible taking into account that our stars are all nearby objects. These $(B-V)$ were taken as $(B-V)_{0}$ and from them, using the temperature-colour scale also given by Arp (1958), effective temperatures were deduced. The distances involved are spectroscopic parallaxes and the adopted spectral types are in Chalonge's system (1952), when available; otherwise, we accepted the most probable in the literature (Jaschek et al. 1964). All related data are collected in columns 6 and 7 of Table I.

Figure 2 shows $D_{P}$ against $\theta=5040 / T$ for the model atmospheres computed by 


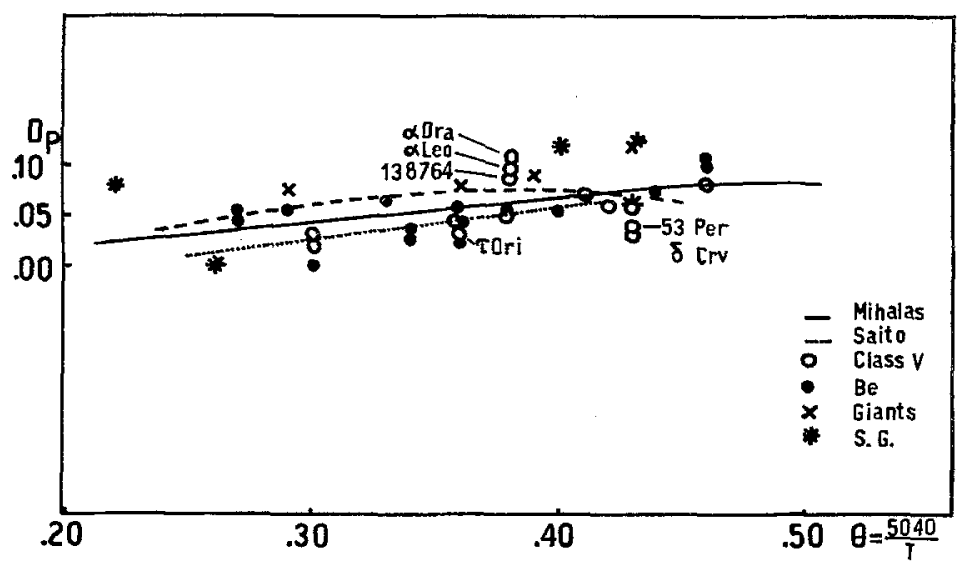

Fig. 2. The three main sequence stars with large $D_{P}$ value deserve a comment; HD 138764 is a class IV object, $\alpha$ Leo is the only main sequence star - in our group - with high rotational velocity and $\alpha$ Dra is a peculiar object in the sense that the Chalonge's system locates it, beyond any doubt, in class $\mathrm{V}$ but according to the M.K.K. system belong, beyond any doubt, to the giants and therefore, its position would not be surprising.

Saito (1956) and Mihalas (1964) - in both sets the Rosseland mean opacity coefficient was used. Our values are shown there (some extremely reddened super giants and $\pi$ Aqri* were left aside); luminosity class $\mathrm{V}$ and Be stars are corrected to take care of the absorption wings effect. The dotted line represents the mean deviation due to a rotational velocity at the limit of stability, through different $\sin (i)$, as deduced from the work of Hardorp and Strittmatter (1968). In Figure 2 it appears that main sequence and Be stars agree with predictions by L.T.E. models. Under this assumption, to lower surface gravity - in the range $0.20 \leqslant \theta \leqslant 0.42$ - lower $D_{P}$ values should be expected, as it appears to be the case in $\tau$ Ori and 53 Per, both stars displaying characteristics of class III objects. $y$ Cor is not a suitable object to discuss because it is the only case in which $\nabla a$ is large and therefore we cannot rely upon the value. We also observe that some Be stars fall on the dotted line. The rotational velocity of these objects justifies, exactly, their location; that means that they hold the necessary but not sufficient condition $v_{0} \sin \left(35^{\circ}\right) \leqslant v \operatorname{rot} \leqslant v_{0} \sin \left(55^{\circ}\right)$, with $v_{0}=390 \mathrm{~km} / \mathrm{sec}$.

Giants and supergiants do not fit in this scheme under L.T.E. conditions. Nor the present calculations following non-L.T.E. approximations approach the actual values. To show this numerically we present, in Table III, the ratio $\phi=D_{P} / D_{B}$ - which is not affected either by rotational velocity or reddening - for stars for which $D_{B}$ was available. The $D_{P}$ used for the main sequence stars are corrected values.

This table can be compared with Table IV which represents the $\phi$ values computed from Mihalas' work (1967). Values in both tables match approximately in the upper left region. The rest is far from agreement. This discrepancy becomes larger as the dimensions of the atmospheres increase. The present state of non-L.T.E. compu-

* See Münch and Lirin (1961). 
TABLE III

$D_{P} / D_{B}$ ratio (corrected)

\begin{tabular}{|c|c|c|c|c|c|c|c|c|}
\hline \multirow{2}{*}{$\begin{array}{l}\text { Spectral } \\
\text { range }\end{array}$} & \multicolumn{8}{|c|}{ Luminosity class } \\
\hline & $\overline{\mathrm{V}}$ & $\begin{array}{l}\text { No } \\
\text { obj }\end{array}$ & $\mathrm{Be}$ & $\begin{array}{l}\text { No } \\
\text { obj }\end{array}$ & III & $\begin{array}{l}\text { No } \\
\text { obj }\end{array}$ & SG & $\begin{array}{l}\text { No } \\
\text { obj }\end{array}$ \\
\hline $\mathrm{A} 0-\mathrm{B} 5$ & $0.13 \pm 0.02$ & 7 & $0.17 \pm 0.02$ & 7 & $0.32 \pm 0.04$ & 3 & $0.40 \pm 0.06$ & 3 \\
\hline B5-B0 & & & $0.32 \pm 0.06$ & 7 & 0.47 & 1 & $0.89 \pm 0.26$ & 6 \\
\hline$>\mathrm{B} 0$ & \multicolumn{8}{|c|}{$1.49 \pm 0.57(3 \mathrm{obj})}$. \\
\hline
\end{tabular}

TABLE IV

$D_{P} / D_{B}$ from Mihalas (1967)

\begin{tabular}{lllllll}
\hline$\theta$ & $\log g$ & & & & & \\
\cline { 2 - 7 } & 4.5 & 4.0 & 3.5 & 3.0 & 2.0 & 1.0 \\
\hline 0.500 & & 0.15 & & 0.16 & 0.16 & 0.04 \\
0.320 & 0.16 & 0.17 & 0.17 & & & \\
0.210 & 0.18 & 0.18 & 0.17 & & & \\
\hline
\end{tabular}

tations (Strom and Kalkofen, 1967; Mihalas, 1967) does not appear to give the solution; we do not know whether the presently obtained coincidence of the population numbers, $n_{1}, n_{2}$, etc. obtained under L.T.E. and non-L.T.E. conditions is a consequence of the ad-hoc hypothesis introduced in non-L.T.E. computations. Whatever the solution may be, our $\phi$ values will provide an interesting test for any new theoretical approach.

Before concluding we may quote that, even though a discussion involving single values of the discontinuity is not adequate - considering the weight of each determination - it will be worth while to revise whether or not a small percentage of giants (in the M.K.K. system) appear with $\mathrm{D}_{P}$ value smaller than that corresponding to class $\mathrm{V}$ objects $-31 \mathrm{Peg}, 53 \mathrm{Per}, \tau$ Ori. We wonder if there is any relation with this feature and the fact that two supergiants from our group $-\varepsilon$ Ori and $\chi^{2}$ Ori - present no discontinuity.

It is opportune to comment that if we adopt, exclusively, the M.K.K. system of spectral classification, our results do not change.

\section{Acknowledgments}

It is a pleasure to acknowledge the Institut d'Astrophysique de Liège for the hospitality during the completion of this work, Dr. L. Houziaux for providing me with most of the spectrograms and tracings and for many helpful discussions, Mme. Andrillat for her permanent collaboration, Dr. E. Mendoza for useful comments and Dr. J. Sahade for revising the manuscript. 


\section{References}

Arp, H. C.: 1958, Handbuch der Physik, Vol. LI, Springer-Verlag, Berlin, p. 83.

Batten, A. H.: 1967, Publ. Dominion Astrophys. Obs. Victoria XIII, 8.

Bloch, M. and Mao-Lin: 1956, Publ. Obs. Lyon 3, 25.

Boyarchuk, A. A. and Kopylov, J. M.: 1964, Publ. Astrophys. Obs. Krimea XXXI, 44.

Chalonge, D. and Divan, L.: 1952, Ann. Astrophys. 15, 20.

Hardorp, J. and Strittmatter, P. A.: 1968, Astrophys. J. 151, 1057.

Jaschek, C., Conde, H., and Sierra, A.: 1964, Serie Astron. XXVIII (2) Obs. Astron. La Plata.

Mianes, P.: 1963, Ann. Astrophys. 26, 1.

Mihalas, D.: 1964, Astrophys. J. Suppl. Ser. XI, 321.

Mihalas, D.: 1967, Astrophys. J. 149, 169.

Münch, G. and Lirin, H.: 1961, Astrophys. J. 133, 11.

Oke, J. B.: 1964, Astrophys. J. 140, 689.

Oke, J. B. and Conti, P. S.: 1966, Astrophys. J. 143, 134.

Saito, S.: 1956, Contr. Inst. Astrophys. Kwasan Obs., Univ. Kyoto, No. 69.

Strom, S. E. and Kalkofen, W.: 1967, Astrophys. J. 149, 191. 